(C) 2015, Elsevier. Licensed under the Creative Commons Attribution-NonCommercialNoDerivatives 4.0 International http://creativecommons.org/licenses/by-nc-nd/4.0/ 


\title{
Analytical and physicochemical characterisation of the senile cataract drug dipeptide $\beta$-alanyl-L-histidine (carnosine)
}

\author{
Hamdy Abdelkader ${ }^{1,3}$, Julian Swinden ${ }^{1}$, Barbara K. Pierscionek ${ }^{2}$ and Raid G Alany ${ }^{1,4}$ \\ ${ }^{1}$ Drug Discovery, Delivery and Patient (DDDPC) Theme, School of Pharmacy and Chemistry, Kingston University London, \\ Kingston upon Thames, London, UK; ${ }^{2}$ Vision Cognition and Neuroscience Theme, Faculty of Science, Engineering and \\ Computing, Kingston University London, Kingston upon Thames, London, UK, ${ }^{3}$ Department of Pharmaceutics, Faculty of \\ Pharmacy, Minia University, Mina, Egypt, ${ }^{4}$ School of Pharmacy, The University of Auckland, Auckland, New Zealand.
}

\section{Abstract}

This study presents a simple but sensitive HPLC chromatographic method with a stabilityindicating assay for determination and physicochemical characterisation of L-carnosine, a promising senile cataract prophylactic agent. Chromatographic analysis was conducted using a reverse phase (RP)-HPLC system and an isocratic mobile phase of $98 \% \mathrm{v} / \mathrm{v}$ trifluoroacetic acid $(0.1 \% \mathrm{v} / \mathrm{v})$ and $2 \% \mathrm{v} / \mathrm{v}$ acetonitrile with detection at $220 \mathrm{~nm}$. L-carnosine was subjected to stress conditions to force its degradation using chemical and thermal agents and was subsequently detected from its degradation products using ESI-MS. The lipophilicity of the drug and 1:1 drug to phospholipid complex (PC) $\mathrm{mol} / \mathrm{mol}$ was determined by estimating the partition coefficient $(P)$. Lipophilicity was greatly enhanced when L-carnosine was formulated as a phospholipid complex using the solvent evaporation method. L-carnosinephospholipid complex could be a promising approach for effective delivery to the human lens as offers a potential novel treatment for senile cataract.

\section{Key words}

L-carnosine, senile cataract, histidine, dipeptide, ocular delivery 


\section{Correspondence}

Hamdy Abdelkader, School of Pharmacy and Chemistry, Kingston University London, UK; h.abdelkader@kingston.ac.uk, ,+447440702427

\section{Introduction}

L-carnosine is an endogenous dipeptide ( $\beta$-alanyl-L-histidine) found in skeletal muscles and nervous tissues in concentrations of 2-25 mM [1, 2]. Human plasma levels of L-carnosine have been reported to rise from levels that cannot be detected to $32 \mu \mathrm{g} / \mathrm{ml}$ as a result of meat consumption [2]. L-carnosine has been found to be a bioactive dipeptide; it possesses antiageing properties which may have application in the treatment of senile cataract [3, 4]. The exact mechanism of how L-carnosine exerts these therapeutic benefits is not clearly known, but it has been suggested that the mechanism can be attributed to potential antioxidant properties and the ability to scavenge free radicals accumulated as a consequence of ageing [5]. This role has yet to be confirmed. It has also been proposed that L-carnosine has a potential anti-gylcation effect, inhibiting or reducing the formation of advanced glycation end products responsible for age-related conditions such as Alzheimer’s [6], skin wrinkling [3] and senile cataracts [4]. Glycation end products result when glucose/reducing sugar moieties react to form amide bonds with the amino groups of proteins (e.g. those found in myelin, collagen and crystallins). The dipeptide carnosine has been found to inhibit the formation of protein carbonyls and cross-linking induced by reducing sugars and reactive aldehyde species, a mechanism termed carnosinylation [7]. The role of L-carnosine in reversing or delaying the onset of senile cataracts is a promising area for further research [8]. Similar to histidine dipeptides, L-carnosine has very weak chromophores that would hamper direct and sensitive spectrophotometric detection. Histidine dipeptides have to be coupled with dyes such as p-bromoaniline and 2,4-dinitro-1-fluorobenzene to form coloured derivatives to allow quantitative detection [9]. However, this approach lacks the sensitivity and reproducibility 
needed for routine analysis. Reverse phase-HPLC methods were developed to quantify Lcarnosine using $100 \mathrm{mM}$ phosphate buffer, $\mathrm{pH} 2$ as mobile phase and UV detection at $210 \mathrm{~nm}$ $[10,11]$. These conditions are problematic as they can shorten the life span of the reversedphase column system used, since it is devoid of any organic phase (e.g. acetonitrile) and the low $\mathrm{pH}$ of 2 coupled with a high inorganic salt concentration (100 mM of phosphate buffer) is likely to block ferules, automatic samplers and be detrimental to the HPLC system and hence produce poor yields. This problem has been tackled with the advent of novel polymeric based supports that graft the reverse hydrophobic moieties onto stationary phase rendering it more resistant to acidic conditions.

In this study, a simple, sensitive, stable and rapid HPLC method was developed and validated according to the guidelines of International Conference on Harmonisation (ICH). The physicochemical properties of L-carnosine, in particular its chemical stability in solution and partition coefficient were investigated. The ability of the dipeptide drug (L-carnosine) to form a complex with a phospholipid in an attempt to alter its n-octanol / water partition coefficient and potentially improve its trans-corneal flux was investigated.

\section{Materials and methods}

\subsection{Materials}

L-carnosine and L-histidine were purchased from Sigma-Aldrich, Dorset, England, the UK. $\beta$-alanine was purchased from BDH Biochemicals, Poole, England. Lipoid S75 was donated by Lipoid GmbH, Ludwigshafen, Germany. 1,4-dioxane and hydrogen peroxide were purchased from Fisher Scientific, Loughborough, UK. All other chemical and reagents were of analytical grade and used as received. 


\subsection{High performance liquid chromatography (HPLC) method development}

An HPLC system (Shimadzu LC-2010AHT, Shimadzu corporation, Kyoto, Japan)

comprising a quaternary pump, an automatic sampler and a UV detector with data acquisition was used. The chromatographic separation was achieved using a Supelcosil C18 column (5 $\mu \mathrm{m} ; 250 \mathrm{~mm} \times 4.6 \mathrm{~mm}$, Supelco Corporation, PA, USA) maintained at $40^{\circ} \mathrm{C}$. The mobile phase comprised trifluoroacetic acid (TFA) (0.1\%) (98\% v/v) and acetonitrile (2\% v/v). The isocratic flow rate was $1.0 \mathrm{ml} / \mathrm{min}$ and the injection volume was fixed at $30 \mu \mathrm{l}$. Detection was by UV absorbance at a wavelength of $220 \mathrm{~nm}$.

\subsubsection{Validation of the HPLC analytical method}

The method was validated according to International Conference on Harmonisation (ICH) guidelines [12] . Approximately $10 \mathrm{mg}$ of carnosine were accurately weighed, transferred into a $10 \mathrm{ml}$ volumetric flask and dissolved in deionised water to produce a stock solution of $1 \mathrm{mg} / \mathrm{ml}$. Calibration standards were prepared by serial dilution using TFA $(0.1 \% \mathrm{v} / \mathrm{v})$ in the concentration range of 10 to $100 \mu \mathrm{g} / \mathrm{ml}$.

\section{Precision and accuracy}

Intra-day and inter-day variability were determined by repeated injections $(n=5)$ of independent samples of the different validation standards. The validation standard concentrations were 15, 40 and $90 \mu \mathrm{g} / \mathrm{ml}$ representing low, middle and high concentrations respectively. Accuracy was assessed by comparing the predicted concentrations of the validation standard samples with the nominal 15, 40 and $90 \mu \mathrm{g} / \mathrm{ml}$ concentrations.

\section{Limit of detection (LOD) and limit of quantitation (LOQ)}

LOD and LOQ were estimated based on signal-to-noise $(\mathrm{S} / \mathrm{N})$ ratio according to ICH guidelines by multiplying the estimated noise using Lab Solutions software version 5.42 SP5, 
Shimadzu Corporation, Japan. The estimated noise was multiplied by 3.3 and 10 for determination of LOD and LOQ respectively

\subsection{Forced degradation studies}

Carnosine was subjected to stress conditions according to ICH guidelines in order to monitor whether the assay was stability-indicating. The investigated stress conditions were acid (0.1 $\mathrm{M} \mathrm{HCl})$ at $60^{\circ} \mathrm{C}$, base $(0.1 \mathrm{M} \mathrm{NaOH})$ at $60^{\circ} \mathrm{C}$, oxidising agent $\left(2 \% \mathrm{v} / \mathrm{v} \mathrm{H}_{2} \mathrm{O}_{2}\right)$ at room temperature and thermal (PBS pH 7.4) at $60^{\circ} \mathrm{C}[13,14]$. The samples were allowed to cool, were neutralised (for acid and base samples), filtered and then diluted prior to quantitative analysis. All experiments were performed in triplicate.

\subsection{Degradation product identification using electrospray ionisation-mass spectroscopy} (ESI-MS)

To identify peaks of the resultant degradation products, freshly prepared carnosine solution and $\mathrm{NaOH}$-degraded samples were analysed using Waters Micromass, LCT mass spectrometer (Waters Corporation, Lancashire, UK) coupled with a KD Scientific syringe pump (KD Scientific, Holliston, Massachusetts, USA). The samples were diluted to $1 \mu \mathrm{g} / \mathrm{ml}$ and were introduced by direct injection at $180 \mu \mathrm{l} / \mathrm{h}$ into an electron spray ionisation (ESI) source in a positive ionisation mode (capillary voltage -3000V), RF value of 89 and desolvation temperature of $300{ }^{\circ} \mathrm{C}$. Data was acquired for $3 \mathrm{~min}$ over a 50 to $1000 \mathrm{~m} / \mathrm{z}$ range. Spectra were averaged over the 3 min and processed using MassLynx software (Waters Corporation, Lancashire, UK).

\subsection{L-carnosine-phospholipid complex}

Equimolar amounts of L-carnosine and lipoid S75 were dissolved in a hydromethanolic solution (30\% water-70\% methanol), transferred into a $100-\mathrm{ml}$ round bottom flask and 
refluxed for 1 hours at $40^{\circ} \mathrm{C}$ and then the solvent was removed by rotary evaporation in a Buchi rotary evaporator (Buchi, Switzerland) [15].

\subsection{Determination of $n$ - octanol/PBS partition coefficient of carnosine}

The partition of L-carnosine between equal volumes of octanol (organic phase) and PBS at $\mathrm{pH} 7.4$ (aqueous phase) was determined at $35^{\circ} \mathrm{C}$ (ocular surface temperature) as previously mentioned [16]. The drug concentration in the organic phase was determined by subtracting the drug concentration in the aqueous phase before and after distribution using the HPLC method described above. The partition coefficient $(P)$ was calculated using equation 3 [17, 18]:

$P=\frac{C_{o}}{C_{w}}=\frac{C_{\text {initial }}-C_{E}}{C_{E}}$

where $C_{o}$ is the concentration of NTX in n-octanol, $C_{w}$ is the total concentration of carnosine in PBS, and $\mathrm{C}_{\text {initial }}$ and $\mathrm{C}_{\mathrm{E}}$ are the concentration of L-carnosine in PBS initially and at equilibrium respectively.

\section{Results and discussion}

\subsection{HPLC method validation}

The system suitability parameters were found to be within acceptable limits. The retention time of L-carnosine under the experimental conditions was 3.8 min and the peak was well resolved from the formulation excipients. An analytical run time of 8 min was sufficient to ensure that all excipients / contaminants had eluted. The following validation parameters were estimated and evaluated according to the ICH guidelines [12]: 


\subsubsection{Linearity}

The linearity was assessed by ANOVA analysis using GraphPad Instat, 3.05, USA to determine the correlation coefficient $(\mathrm{R})$ and $\mathrm{P}$ value. The standard calibration curve was linear in the range of $10-100 \mu \mathrm{g} / \mathrm{ml}(\mathrm{R}>0.999$ and $\mathrm{P}<0.0001)$. Percentage (\%) recovery $\pm \%$ relative standard deviation (RSD) ranged from $96.2 \pm 4.0$ to $101.7 \pm 1.7$ (Table 1 ). LOD and LOQ were estimated to be $0.5 \mu \mathrm{g} / \mathrm{ml}$ and $1 \mu \mathrm{g} / \mathrm{ml}$ respectively.

\subsubsection{Precision and accuracy}

The precision and accuracy of the method were reported as the relative standard deviation (\%RSD) and \% recovery of the QC samples (Table 2). The intra-day and inter-day precision for the three samples were found to be between 0.4 and $2.87 \%$, and 0.44 and $2.5 \%$ respectively. These values comply with the acceptance criteria of the ICH guidelines [19].

\subsection{Forced degradation studies}

L-carnosine was subjected to a series of forced degradation cycles for 7 days in order to obtain appreciable degradation products that could be resolved on the chromatograms and further reflect the ability of the developed method to differentiate between analyte and degradation product peaks. The analyte peaks were well resolved from peaks for the reagents used and reagent stabilisers $\left(\mathrm{H}_{2} \mathrm{O}_{2}\right)$ as shown in Figure 1. Percentage recovery values of Lcarnosine under the stress conditions are shown in Figure 2. The drug solution showed slight to extensive degradation dependent upon the stress condition used.

Approximately $30 \%$ of the initial concentration of L-carnosine was lost after exposure to strong alkaline conditions for 7 days at $60{ }^{\circ} \mathrm{C}$. Exposure of L-carnosine to a strong acid showed moderate degradation. The extent of L-carnosine degradation in PBS was significantly less ( $\mathrm{P}<0.01$, unpaired t test) than that in the base or acid solutions. Lcarnosine showed slight degradation when subjected to an oxidizing agent. Confirmation of the degradation products was performed by using $1 \mathrm{mg} / \mathrm{ml}$ of pure solution of $\beta$-alanine and 
histidine. The histidine peak eluted at the same retention time of alkaline and acidic degradation products at 3.39 min. However, $\beta$-alanine did not show up on the UV detector due to absence of chromophores. These findings indicate that the assay was stability indicating and the main degradation mechanism of L-carnosine is by hydrolysis of the both peptide bonds forming the constituent amino acids.

\subsection{Degradation product identification using electron spray ionisation-mass} spectroscopy (ESI-MS)

Mass spectra were generated to determine the molecular weight of the drug and main degradation products in solution at $60^{\circ} \mathrm{C}$ under alkaline conditions (Figure 3A and B). Other stressed drug solutions in HCL, PBS and hydrogen peroxide showed insignificant (less than 10\%) degradation as detected with ESI-MS.

The mass spectra were determined on a LCT- Time of Flight (TOF)-MS system, using ESI in negative mode. Under these detection conditions, the sample ionises by interacting with the solvent (in this case acetonitrile). The detector can detect negative ions. The results showed that L-carnosine and two major ion peaks (histidine and alanine) appeared at 225, 154.1 and $88.1 \mathrm{~m} / \mathrm{z}$ respectively. These masses correspond to the negatively charged ion (M-H) of carnosine; and its constituent amino acids suggesting that the mechanism of degradation is by hydrolytic cleavage of both peptide bonds into the comprising amino acids.

\subsection{Partition coefficient (n- octanol/PBS) of carnosine}

Drug lipid solubility (lipophilicity) is an essential property for drug permeation via lipid membranes such as the corneal epithelium [20]. Drug lipophilicity is related to its chemical structure and can be assessed by measuring the partition coefficient $(P)$. The results showed that $P$ values of L-carnosine and L-carnosine-phospholipid complex at $35^{\circ} \mathrm{C}$ (ocular temperature)[21] were 0.026 and 0.55 respectively. The results showed a significant increase 
( $\mathrm{P}<0.001)$ when L-carnosine was formulated as a phospholipid complex. The estimated $P$ value for L-carnosine-phospholipid complex was found to be twenty times greater than that for L-carnosine. L-carnosine is a highly hydrophilic drug and delivery it to the eye requires a penetration enhancer. Using the L-carnosine-phospholipid complex offers a viable alternative.

\section{Conclusion}

This study investigated the chemical stability of L-carnosine under different stress conditions. The main degradation pathway was found to be hydrolysis. Lipophilicity studies showed that L-carnosine is hydrophilic and that its permeation through the cornea would be predominantly dependent on the epithelium barrier. Lipid-based systems such as phospholipid complexes (PC) could improve L-carnosine lipophilicity, impart possible permeation enhancing effects and prolong precorneal residence time. Further investigations into PC as possible ocular carriers for L-carnosine are underway.

\section{References}

[1] K.G. Crush, Carnosine and related substances in animal tissues, Comp. Biochem. Physiol., 34 (1970) 3-30.

[2] Y.J. Park, S.L. Volpe, E.A. Decker, Quantitation of carnosine in humans plasma after dietary consumption of beef, J. Agric. Food. Chem., 53 (2005) 4736-4739.

[3] A.R. Hipkiss, Would carnosine or a carnivorous diet help suppress aging and associated pathologies?, Ann N Y Acad Sci., 1067 (2006 ) 369-374.

[4] T. Toh, J. Morton, J. Coxon, M. Elder, Medical treatment of cataract, Clin Experiment Ophthalmol. , 35 (2007) 664-671. [5] M.A. Babizhayev, L. Burke, P. Micans, S.P. Richer, N-Acetylcarnosine sustained drug delivery eye drops to control the signs of ageless vision: Glare sensitivity, cataract amelioration and quality of vision currently available treatment for the challenging 50,000-patient population, Clin Interv Aging., 4 (2009) 31-50.

[6] N. Sasaki, F. R., K. Tsuzuki, Y. Hayashi, T. Yoshida, N. Fujii, T. Koike, I. Wakayama, R. Yanagihara, R. Garruto, N. Amano, Z. Makita, Advanced glycation end products in Alzheimer's disease and other neurodegenerative diseases, Am. J. Pathol., 153 (1998) 1149-1155.

[7] A.R. Hipkiss, C. Brownson, M.J. Carrier, Carnosine, the anti-ageing, anti-oxidant dipetide, may react with protein carbonyl groups, Mech. Ageing Dev. Sep 15;(13). 122 (2001) 1431-1445.

[8] H. Abdelkader, R.G. Alany, B. Pierscionek, Age-related cataract and drug therapy: opportunities and challenges for topical antioxidant delivery to the lens, J. Pharm. Pharmacol., 67 (2015) 537-550.

[9] C.P. Parker, Spectrophotometric determination of carnosine, anserine, and taurine in skeletal muscle, Anal. Biochem., 108 (1980) 303-305.

[10] C.A. Chrinchton, C. Lamont, D.J. Miller, Detection and estimation of carnosine, anserine and novel imidazoles in cardiac and skeletal muscles in several species by high performance liquid chromatography, J. Physiol., 398 (1988) 74. 
[11] J.J. O'Dowd, D.J. Robins, D.J. Miller, Detection, characterisation, and quantification of carnosine and other histidyl derivatives in cardiac and skeletal muscle, Biochim. Biophys. Acta, 967 (1988) 241-249.

[12] ICH(Q2), Validation of analytical procedures: text nd methodology Q2 (R1), (November 2005).

[13] D.W. Reynolds, K.L. Facchine, J.F. Mullaney, K.M. Alsante, T.D. Hatajik, M.G. Motto, Available guidance and best practices for conducting forced degradation studies, Pharm. Technol., February (2002) 48-56.

[14] K.M. Alsante, L. Martin, S.W. Baertschi, A stress testing benchmarking study, Pharm. Technol., February (2003) 60-72.

[15] M.S. Freag, Y. Elnaggar, O.Y. Abdallah, Lyophilized phytosomal nanocarriers as platforms for enhanced diosmin delivery: optimization and ex vivo permeation, Int J Nanomedicine, 8 (2013) 2385-2397.

[16] H. Abdelkader, Z. Wu, R. Al-Kassas, J. Brown, R. Alany, Preformulation characteristics of the opioid growth factor antagonist-naltrexone hydrochloride: stability and lipophilicity studies, Journal of Drug Delivery Science and Technology, 21 (2011) 157-163.

[17] A.T. Florence, D. Attwood, The solubility of drugs, in: A.T. Florence, D. Attwood (Eds.) Physichochemical Principles of Pharmacy, McMillan Press Ltd, London, 1998, pp. 152-197.

[18] Z. Wu, I.G. Tucker, M. Razzak, N. Medlicott, Physichochemical characterisation of ricobendazole: I. solubility, lipophilicity and ionization charactersitics, J Pharm Sci, 94 (2005) 983-993.

[19] V.P. Shah, K.K. Midha, S. Dighe, I.L. Mcgilveray, J.P. Skelly, A. Jacobi, Analytical methods validation: Bioavailability, bioequivalence, and pharmacokinetic studies J. Pharm. Sci. , 81 (1992) 309-312.

[20] R.D. Schoenwald, R.L. Ward, Relationship between steroid permeability across excised rabbit cornea and octanolWater partition coefficients, J. Pharm. Sci., 67 (1978) 786-788.

[21] R.D. Schoenwald, H.S. Huang, Corneal penetration behavior of beta blocking agents I: physichochemical factors, J. Pharm. Sci, 72 (1983) 1266-1272. 


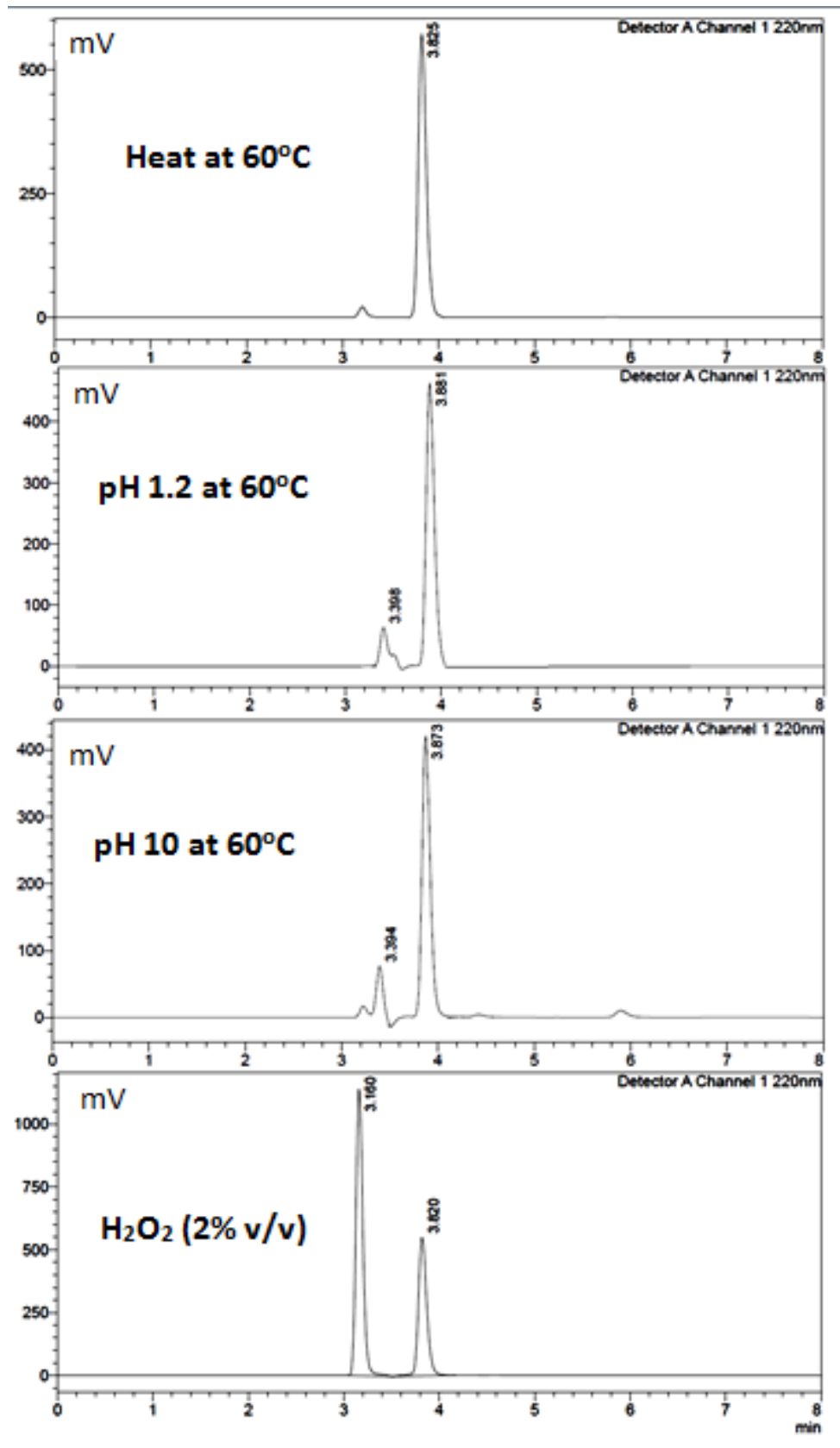

Figure 1 HPLC chromatograms of L-carnosine after exposure to stress conditions of heat (at 60 for 7 days), acid (0.1 M HCl at 60 for 7 days), alkaline (0.1 M Na OH at 60 for 7 days) and hydrogen peroxide (2\% at ambient conditions for 24 hours). 


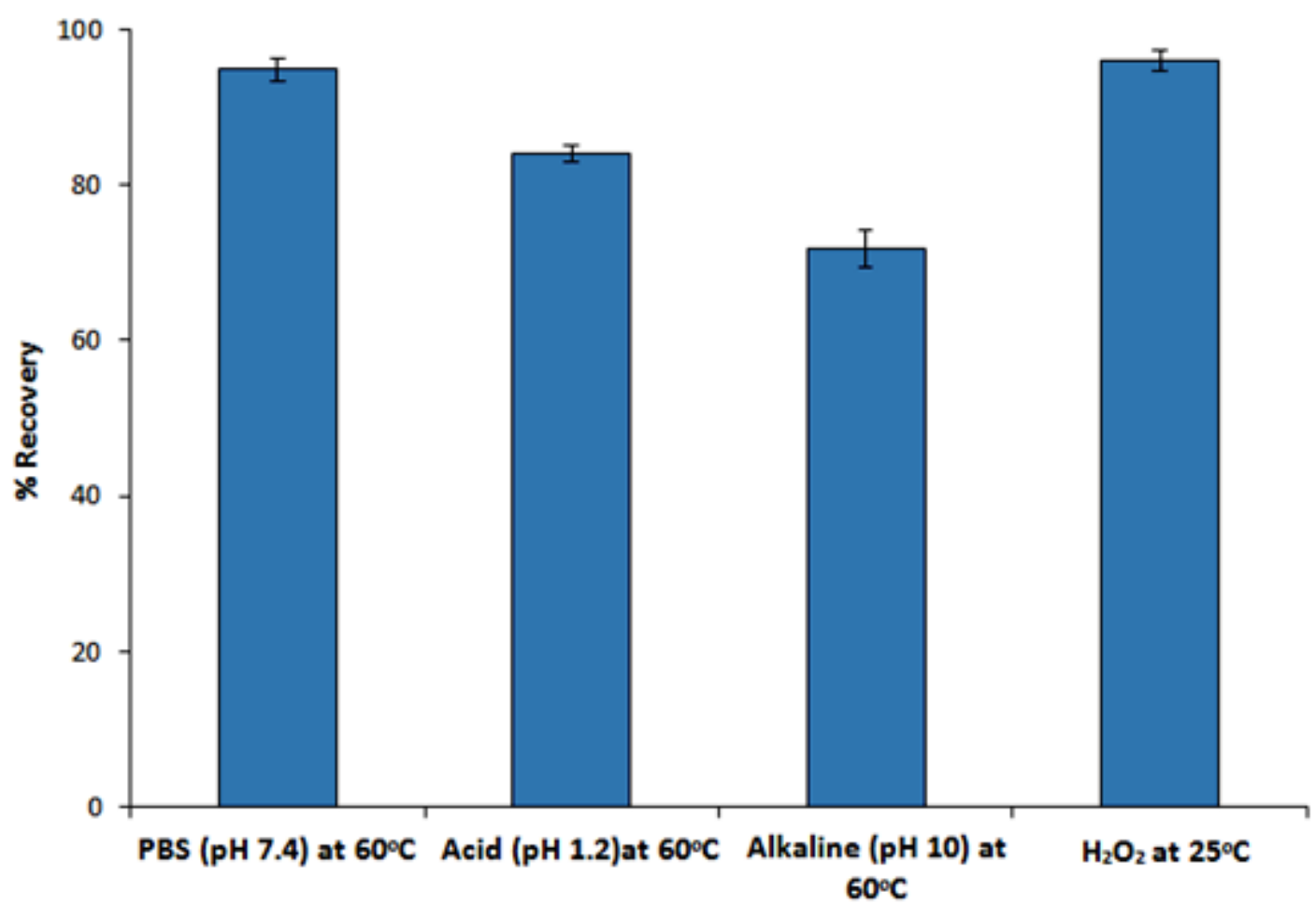

Figure 2 Percentage (\%) recovery of L-carnosine after exposure to various stress conditions 
(A)

resh carnosine swept 89s25:

HuM Dio7nov-910 5 (0260) Cm (3.5)

100 . 1250

\begin{tabular}{l|l}
${ }^{100}$ & $\begin{array}{r}\text { Carnosine } \\
\text { [MN-1] }\end{array}$
\end{tabular}

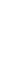
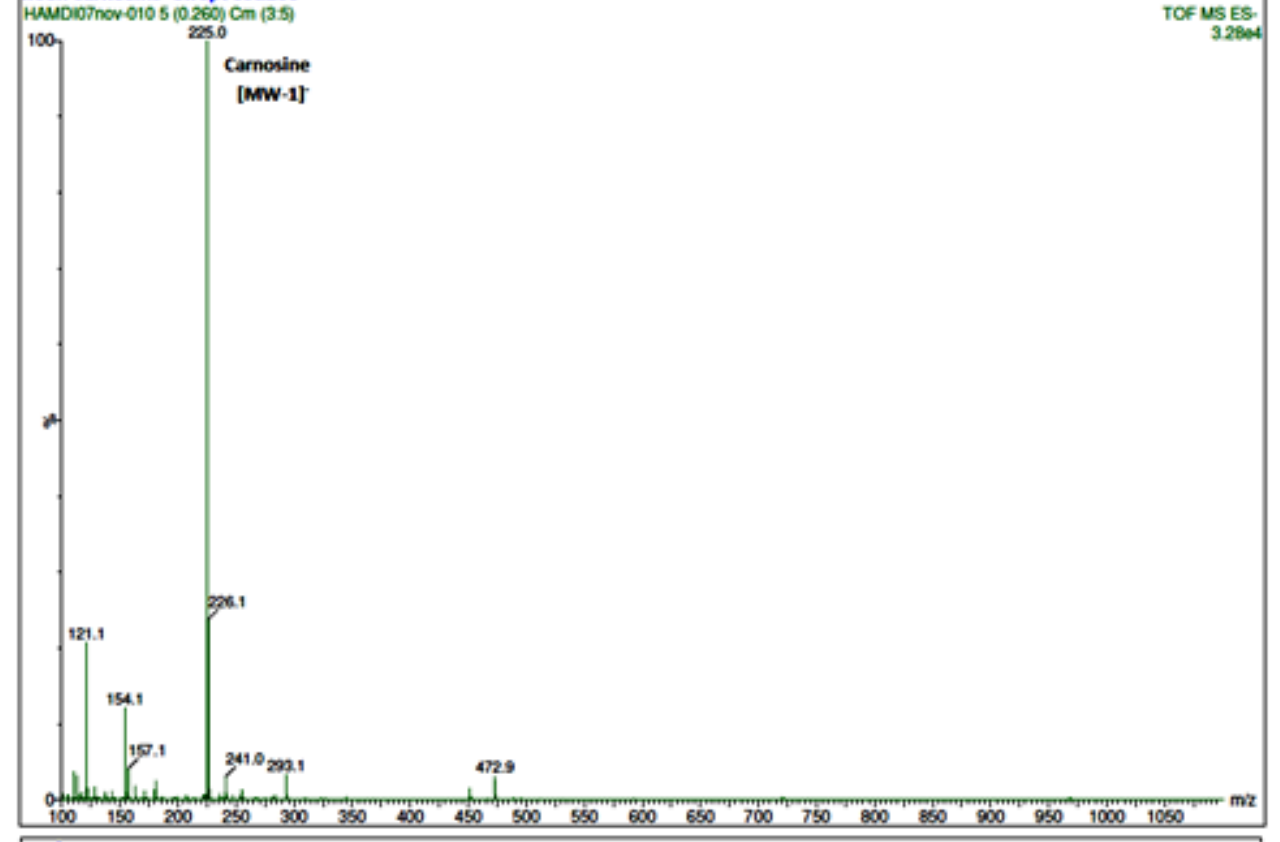

(B)

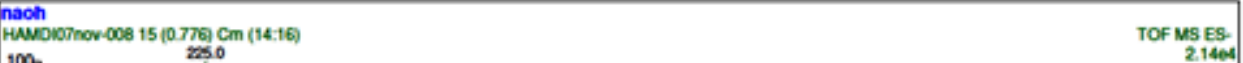

HWWONOTnow.008 15 (0.776 Cm (14:16)

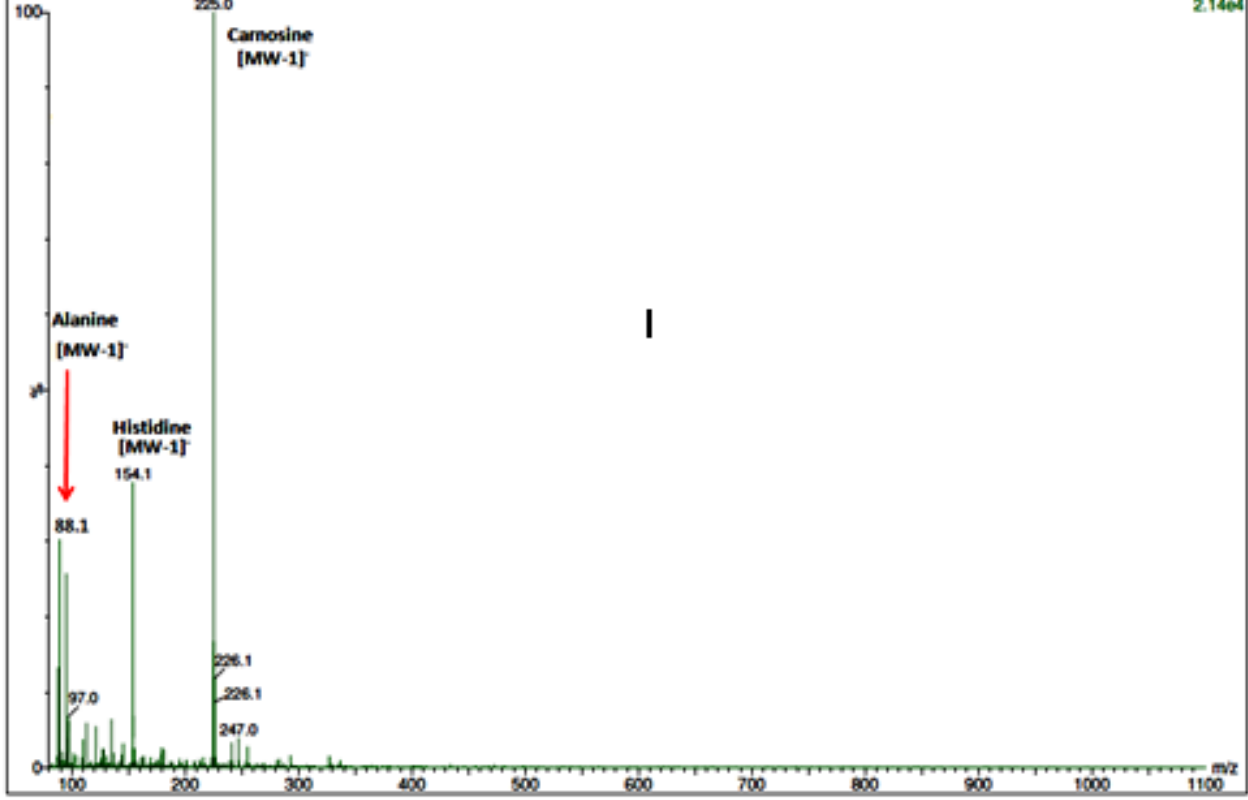

Figure 3 ESI MS spectra for L-carnosine and canrosine and its degradation products (B) 
Table 1 Predicted concentrations, \% recovery and linear regression parameters for the developed analytical method

\begin{tabular}{|c|c|c|c|c|c|c|c|c|}
\hline \multirow[b]{2}{*}{ Set number } & \multicolumn{5}{|c|}{ Nominal concentration $(\mu \mathrm{g} / \mathrm{ml})$} & \multirow[b]{2}{*}{$\mathrm{R}$} & \multirow[b]{2}{*}{ Slope } & \multirow[b]{2}{*}{ Intercept } \\
\hline & 10 & 20 & 50 & 80 & 100 & & & \\
\hline 1 & 9.81 & 20.59 & 51.91 & 81.43 & 101.20 & 0.9998 & 34891 & 43147 \\
\hline 2 & 9.96 & 21.05 & 51.67 & 80.57 & 101.06 & 0.9998 & 34587 & 54529 \\
\hline 3 & 8.78 & 18.79 & 50.47 & 80.07 & 100.19 & 0.9998 & 34989 & -7651 \\
\hline 4 & 9.78 & 19.41 & 50.14 & 80.15 & 96.05 & 0.9981 & 33486 & 51256 \\
\hline 5 & 9.65 & 19.29 & 50.04 & 79.95 & 98.017 & 0.9991 & 34072 & 30212 \\
\hline Mean & 9.62 & 19.83 & 50.85 & 80.43 & 99.31 & 0.9994 & 34405 & 34299 \\
\hline \% RSD & 4.00 & 3.50 & 1.70 & 0.75 & 1.90 & & & \\
\hline \% recovery & 96.20 & 99.15 & 101.70 & 100.50 & 99.31 & & & \\
\hline
\end{tabular}


Table 2 Precision and accuracy of the validation standard (Results are expressed as mean values, $n=5$ )

\begin{tabular}{c|ccc|ccc}
\hline \multirow{2}{*}{} & \multicolumn{5}{|c}{ Nominal concentration $(\mu \mathrm{g} / \mathrm{ml})$} \\
\cline { 2 - 7 } & \multicolumn{3}{|c}{ Intra-day } & \multicolumn{3}{c}{ Inter-day } \\
\cline { 2 - 7 } & 15 & 40 & 90 & 15 & 40 & 90 \\
\hline Mean & 14.55 & 40.10 & 89.40 & 14.35 & 38.80 & 88.50 \\
\% RSD & 2.87 & 1.26 & 0.40 & 2.5 & 1.5 & 0.44 \\
\% Recovery & 97.00 & 100.25 & 99.33 & 95.66 & 97.00 & 98.33 \\
\hline
\end{tabular}

